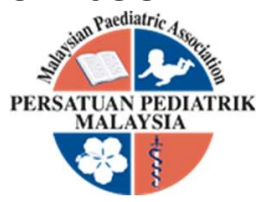

\title{
SILENT MASTOIDITIS ASSOCIATED WITH PNEUMOCOCCAL MENINGITIS
}

\author{
Ashikin Mohd Nordin', Ong Jun Jean', Juriza Ismail ${ }^{3}$, Norazlin Kamal Nor ${ }^{3}$, Wong Sau Wei ${ }^{3}$, Noor Dina \\ Hashim ${ }^{4}$, Fahrin Zara Mohammad Nasseri', Adibah Abdul Ghafar ${ }^{3}$, Erica Yee Hing ${ }^{5}$, Nurdiyana \\ Nasrudin $^{6}$, Asma Abdullah ${ }^{4}$
}

\begin{abstract}
Streptococcus pneumoniae (S pneumoniae) can cause a wide spectrum of diseases which includes upper respiratory tract infection as well as more severe invasive disease such as meningitis. Meningitis may be caused by invasion of the organism through the blood brain barrier, either via haematological spread or from an adjacent focus of infection such as the ears. We describe two infants with pneumococcal meningitis and silent mastoiditis. They both presented with a classical history to suggest meningitis with no apparent focus of infection. A brain imaging was done in the first infant to look for the underlying cause of his focal seizure and in the second infant, to assess for complications of meningitis, as he had a slow recovery. While they did not have any clinical signs to point towards the diagnosis, they were both diagnosed to have acute mastoiditis from brain imaging. We would like to highlight the importance of brain imaging in excluding silent mastoiditis in infants with meningitis, particularly in those whose clinical course appears atypical.
\end{abstract}

Keywords: Pneumococcal Meningitis, Silent Otitis Media, Mastoiditis, Meningitis

DOI: 10.51407/mjpch.v27i2.118

\section{Introduction}

Pneumococcal meningitis in children has approximately $8 \%$ mortality rate [1]. It can be caused by invasion of the organism through the blood brain barrier, which may be due to an anatomical defect or an adjacent primary focus, or through haematological spread. The most common primary focus of infection is the ear (30\%), followed by the lung (18\%), sinus ( $8 \%)$, others (2\%), and in some, an unidentified primary focus (42\%) [2]).

Acute mastoiditis (AM) presents with either signs of acute otitis media (AOM) and local inflammatory findings over the mastoid area with radiological or surgical findings of mastoiditis $[3,4]$. AM is less prevalent in younger children below 6 months of age and is commonly preceded by upper respiratory infection and clear clinical signs suggestive of AM [5]. We report two
Received: 28 September 2020; Accepted revised manuscript: 28 April 2021

Published online: 19 July 2021 young infants with pneumococcal meningitis whose signs of AM were not clinically apparent, with one of the infants having been diagnosed with a rotavirus infection just prior to the diagnosis of pneumococcal meningitis.

\footnotetext{
${ }^{1}$ Faculty of Medicine, University of Cyberjaya, Selangor, Malaysia ${ }^{2}$ Faculty of Medicine, International Medical University, Bukit Jalil, 57000 Kuala Lumpur, Malaysia

${ }^{3}$ Department of Paediatrics, Universiti Kebangsaan Malaysia, 56000 Cheras, Kuala Lumpur, Malaysia.

${ }^{4}$ Department of Otorhinolaryngology, Universiti Kebangsaan Malaysia, 56000 Cheras, Kuala Lumpur, Malaysia.

${ }^{5}$ Department of Radiology, Universiti Kebangsaan Malaysia, 56000 Cheras, Kuala Lumpur, Malaysia.

${ }^{6}$ Faculty of Medicine, Universiti Teknologi MARA, 47000 Jalan Hospital, Sungai Buloh, Selangor, Malaysia

Corresponding Author:

Dr. Ashikin Mohd Nordin, Faculty of Medicine, University of Cyberjaya, Persiaran Bestari 11, 63000, Cyberjaya, Selangor Tel.: +603-83137000 Email: ashikin@cyberjaya.edu.my, drashikinmn@gmail.com
} 


\section{Case 1}

A 5-month-old infant presented with three days of fever, reduced activity and left clonic seizures on the day of admission. This was preceded by a rotavirus gastroenteritis three days prior. He was not septic clinically and had no focal neurological signs. Systemic examination, including otoscopic examination was normal. The initial working diagnosis was meningitis with a differential of Herpes simplex encephalitis. High dose intravenous (IV) crystalline penicillin (C-Pen), IV cefotaxime and IV acyclovir were started empirically on admission. His blood cultures were clear however his cerebrospinal fluid (CSF) examination confirmed a pneumococcal meningitis with high protein $(1992 \mathrm{mg} / \mathrm{dL})$ and low CSF: plasma glucose ratio (28\%) as well as a positive $S$ pneumoniae culture from his CSF.

A brain computed tomography (CT) scan was done on the following day to elucidate the cause of his focal seizure. CT brain showed a normal brain structure with presence of mastoid opacification and this was supported by his brain magnetic resonance imaging (MRI) findings (Figure 1). Surprisingly, a repeat otoscopic examination revealed a normal tympanic membrane without posterior wall sagging.

Despite medical treatment, the child still had seizures. Based on the clinical and radiological evidence we hypothesized that the mastoiditis could be a possible source of his intracranial infection and focal seizures. Thus, a mastoidectomy was performed after 7 days of high dose antibiotics. Intraoperative findings supported our hypothesis: there was granulation tissue in the mastoid air cells area. His fever and focal seizures abated after surgery and he fully recovered without obvious neurological sequelae upon discharge. He completed 6 weeks of high dose IV C-Pen and IV cefotaxime.

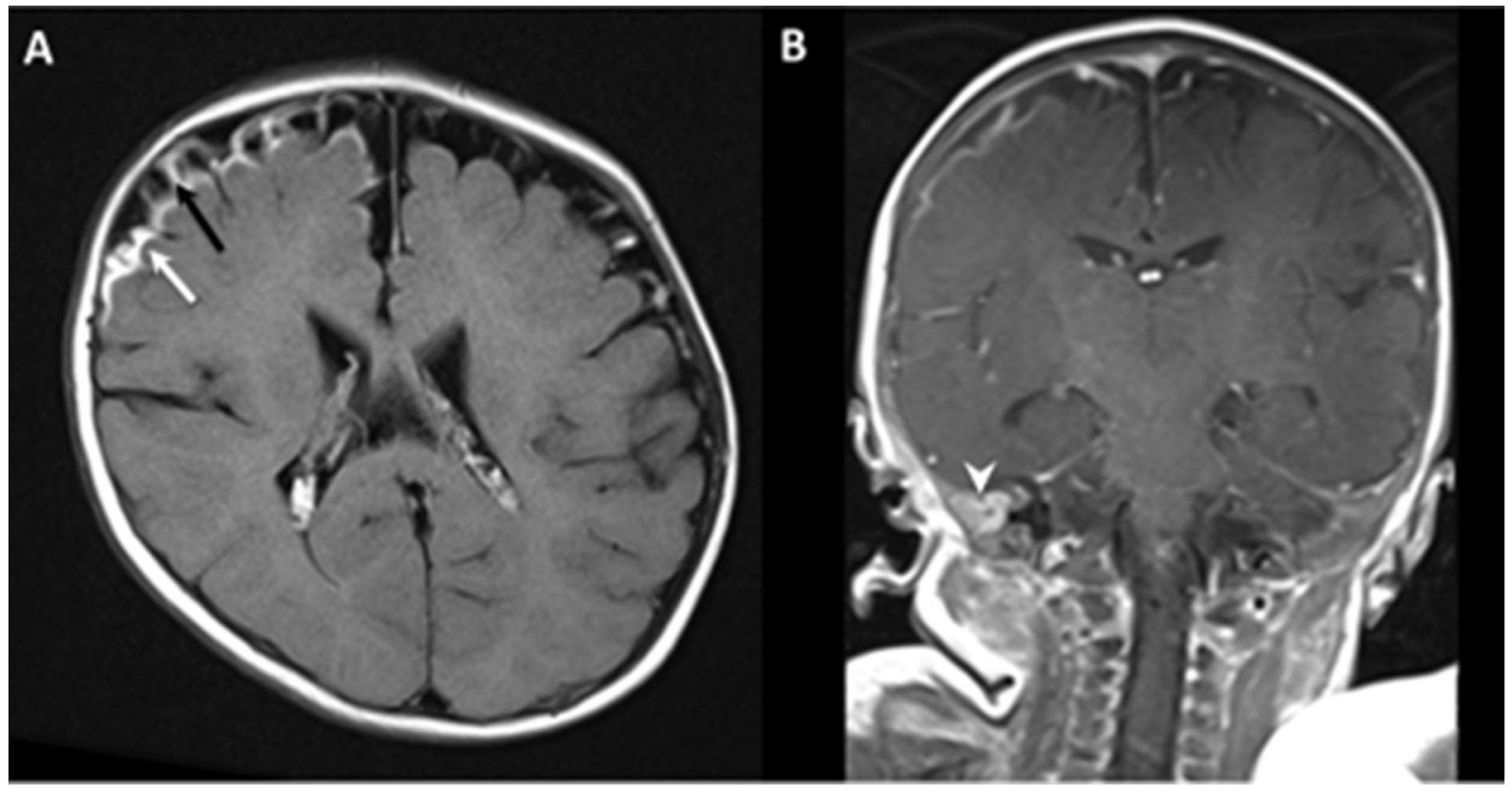

Figure 1. MRI brain

A: T2 FLAIR axial post contrast sequence showing leptomeningeal enhancement (white arrow) and thick enhancing exudates along vessels that transverse the frontal subarachnoid space bilaterally (black arrow). B: T1 coronal post contrast sequence showing enhancement within right mastoid air cells (white arrowhead) and right middle ear, suggestive of otitis media. 


\section{Case 2}

A 4-month-old infant presented with generalised seizures after four days of high-grade fever associated with upper respiratory tract infection symptoms, diarrhoea and vomiting. He was fully breastfed and was vaccinated according to the national healthcare schedule. Initial examination did not reveal any focal neurological signs or clinical suspicion of otitis media and mastoiditis. Intubation and cerebral protection were required due to deteriorating consciousness. He was suspected to have bacterial meningitis and empirically treated with high dose IV C-Pen and IV cefotaxime. CSF examination confirmed pneumococcal meningitis with a high CSF protein (17063mg/dL) and low CSF plasma:glucose ratio (29\%) and a positive latex agglutination test for $S$. pneumoniae. His blood cultures were clear.
Unfortunately, his general condition did not improve after 48 hours, and a brain MRI was performed to look for intracranial complications of meningitis. His MRI brain (Figure 2) showed presence of a dural sinus thrombosis, micro abscesses of the perivascular space and right mastoiditis. Subsequently, repeated otoscopic examination showed red tympanic membrane with prominent blood vessel.

A mastoidectomy was done as he had persistent fever and poor clinical improvement despite adequate antibiotics. Mastoidectomy revealed granulation tissue in the mastoid air cells. His clinical condition improved tremendously after surgery. He completed 6 weeks of IV ceftriaxone and made a full recovery upon discharge.

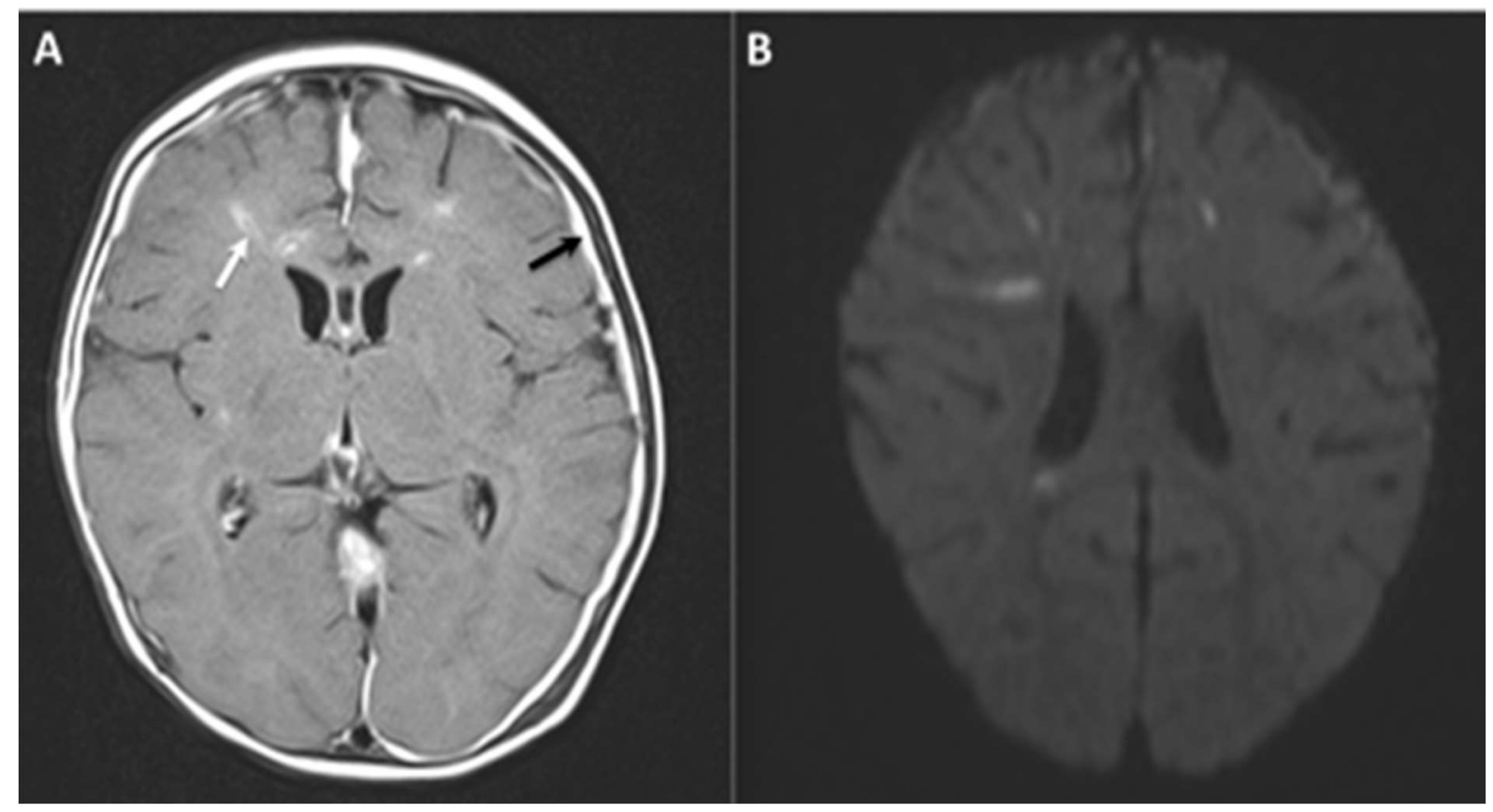

Figure 2. MRI brain

A: T2 FLAIR axial post contrast showing enhancement along dilated Virchow-Robin spaces (white arrow). There is diffuse bilateral pachymeningeal enhancement (black arrow). B: DWI showing restricted diffusion within the dilated Virchow-Robin spaces suggestive of pus within. The pachymeninges also demonstrate restricted diffusion. 


\section{Discussion}

Meningitis typically presents with fever and signs of meningeal inflammation. However, in younger infants, signs of meningism may not be very pronounced. Clinical presentation of pneumococcal meningitis can be variable and non-specific. It is not uncommon for an infant to present with diarrhoea and vomiting preceding more overt symptoms of meningitis [6]. A previous study has also reported that the most common presentation in infants less than 6months-old are fits, irritability, a bulging fontanelle and diarrhoea [7]. Both our infants had diarrhoea preceding the fever. The presence of seizures prompted examination of the CSF and subsequent management of meningitis. One child was moderately septic, however the other infant remained non-toxic for the whole duration of treatment. One of our infants had confirmed rotavirus gastroenteritis three days prior to the diagnosis of meningitis.

Review of literature revealed a limited number of case reports of meningitis or bacteraemia following rotavirus infection in infants, and the exact pathophysiology is not yet known [8]. It is postulated that the mucosal damage caused by rotavirus infection may allow translocation of bacteria from the intestine. It is estimated that bacteraemia after rotavirus infection in children has a prevalence rate of $0.32 \%$ [9]. Other studies have reported an incidence between $0.22 \%$ [10] and $0.35 \%$ [11]. However, prevalence of secondary bacteraemia in infants is postulated to be higher at $0.68 \%$ [11] with the commonest presentation being recrudescence of fever $[8,9]$. Most of the secondary bacteraemia following a rotavirus infection are caused by bacteria from the enterobacteriaceae family such as Klebsiella pneumoniae and Enterobacter chloacae, however Streptococcus viridans as a cause of secondary bacteraemia has also been reported [9]. In our first case, a secondary peak of fever after recovery from a rotavirus infection initially raised the suspicion of a secondary bacteraemia. However, he was ultimately diagnosed to have streptococcal meningitis without associated bacteraemia. We postulate that his clinical presentation of fever and focal seizure could possibly be due to local extension of infection from his silent mastoiditis.
We would like to highlight that both our patients had $A M$ without corresponding clinical signs of AOM or AM. Acute mastoiditis is usually rare among infants below 6-months-old, and if present, its progression is rapid and usually accompanied by clear retro-auricular signs such as protruding ear and redness behind the ear [5]. However, in these two infants, the typical clinical appearance of mastoiditis was not observed.

Silent otitis media was first described by Paparella et al. [12], and subsequent published research has demonstrated that otitis media may be present despite no corresponding changes in the tympanic membrane $[13,14]$. Without timely treatment, otitis media can lead to complications such as acute mastoiditis, and the infection can spread to adjacent structures. Due to developmental immaturity and inability to clearly express symptoms, silent mastoiditis needs to be seriously considered as a diagnosis in young children less than two years of age who present with fever with no clear aetiology [15].

'Silent Mastoiditis' or a diagnosis of acute mastoiditis without accompanying changes in the tympanic membrane or presence of retroauricular sign has also been reported[16]. However, the patients reported were older. One patient was an immunocompetent 8-year-old girl that presented with multiple intracranial complications and another was a 25-month-old girl that presented with facial nerve palsy [17]. All these children did not have accompanying changes in their tympanic membrane. However, in the older children, there was a history to suggest presence of otitis media prior to the onset of the complications, which was not present in our infant. In both our patients, the diagnosis of acute mastoiditis was delayed due to absence of pathologic changes at the tympanic membrane or the retro-auricular area. The diagnosis was made by brain imaging which were indicated for further investigation of focal seizure as well as poor response to antibiotic treatment.

S.pneumoniae is the most common organism causing AOM, and evidence supports the use of pneumococcal conjugate vaccine to reduce the rate of AOM $[18,19]$. Unfortunately, both infants had not received the pneumococcal conjugate vaccine, which despite being strongly recommended in this age group, was not yet in the national immunization schedule. 
In conclusion, our cases highlight the following:

1. Otitis media and mastoiditis may be a source of infection in children with meningitis even when physical findings are minimal, this is especially true in very young infant.

2. A normal otoscopic examination does not exclude mastoiditis.

3. A CT brain is indicated in children who show poor response to adequate antibiotics after 48-72 hours, a deterioration in clinical presentation as well as those with an unexplained cause of a focal seizure or neurological deficit.

4. Though uncommon, secondary bacteraemia after rotavirus infection must be considered in children with recrudescence of fever.

\section{References:}

[1] Aristegui JCJ, Rodrigo C, Jose DL, Martinón M, Fernández C. Clinical data and factors associated with poor outcome in pneumococcal meningitis. European Journal of Paediatrics. 2006;285-9.

[2] Østergaard C, Konradsen HB, Samuelsson S. Clinical presentation and prognostic factors of Streptococcus pneumonia meningitis according to the focus of infection. BMC Infectious Disease. 2005;5:93.

[3] Chesney J, Black A, Choo D. What is the best practice for acute mastoiditis in children? Laryngoscope. 2013;124(5):1057-8.

[4] Ferlito A, Paparella MM, Rinaldo A, Schachern A, Cureoglu S. The entity known as chronic silent ( subclinical) otitis media: a common lesion and a forgotten diagnosis. Acta Otolaryngol. 2003;(12):749-51.

[5] Stenfeldt K, Enoksson F, Stalfors J, Hultcrantz $M$, Hermansson A \&Groth $A$. Infants under the age of six months with acute mastoiditis . A descriptive study of
15 years in Sweden. International Journal of Paediatric Otorhinolaryngology. 2014;78(7):1119-22

[6] Shahrin L, Chisti MJ, Huq S, Islam MM, Alam S. Case Report Diarrhea-associated pneumococcal meningitis with complicating hydrocephalus in a child in a resource-limited setting. J Infect Dev Ctries. 2016;10(8):888-91.

[7] Kirkpatrick B, Reeves DS, Niacgowan AP, Bsio B. A review of the clinical presentation, laboratory features, antimicrobial therapy and outcome of 77 episodes of pneumococcal meningitis occurring in children and adults. J Infect. 1994;29:171-82.

[8] Lowenthal A, Livni G, Amir J, Samra Z, Ashkenazi S. Secondary bacteremia after rotavirus gastroenteritis in infancy. Pediatrics. 2006;117(1):224-6.

[9] Scheinier Eric AS. Septicemia following rotavirus gastroenteritis. Isr Med Assoc J. 2013;15(March 2013):166-9.

[10] Gonzalez-Carretero P, Noguera A FC. Rotavirus gastroenteritis leading to secondary bacteremia in previously healthy infants [Letter to the editor]. Pediatrics. 2006;118(5):2255-6.

[11] Lowenthal A, Livni G AS. Rotavirus gastroenteritis leading to secondary bacteremia in previously healthy infants : in Reply. Pediatrics. 2006;118(5):2255a 6.

[12] Paparella, Michael M., Goycoolea Marcos V., Meyerhoff William L. SD. Endolymphatic hydrops and otitis media. Laryngoscope. 1979; 89:43-54.

[13] Paparella MM, Schachern PA, Cureoglu. S. Chronic silent otitis media. ORI. 2002;64(2):65-72.

[14] Jaisinghani VJ, Paparella MM, Schachern PA, Le CT. Tympanic membrane/middle ear pathologic correlates in chronic otitis media. Laryngoscope. 1999;109(5):712-6.

[15] Baylan Muzeyyan Yildirim, Schachern Patricia, Tsuprun Vladimir, Shiabata Dai, Paparella Michael M CS. The pathology of silent otitis media: a predecessor to tympanogenic meningitis in infants. Int J 
Pediatr Otorhinolaryngol.

2014;78(3):451-4.

[16] Voudouris C, Psarommatis I, Nikas I, Kafouris D, Chrysouli K, Report C. Pediatric masked mastoiditis associated with multiple intracranial complications. Case Rep Otolaryngol. 2015;2015.

[17] Fukuda T, Sugie H, Ito M, Kikawada T. Bilateral facial palsy caused by bilateral masked mastoiditis. Pediatr Neurol. 1998;18(4):351-3.

[18] Taylor S, Marchisio P, Vergison A, Harriague J, Hausdorff WP, Haggard M. Impact of pneumococcal conjugate vaccination on otitis media: a systematic review. Clin Infect Dis. 2012;54(12):176573.

[19] Casey JR, Pichichero ME. Changes in frequency and pathogens causing acute otitis media in 1995-2003. Pediatr Infect Dis J. 2004;23(9):824-8. 